\title{
Evaluation of Serum C24 Ceramide as a Predictor of Hepatic Decompensation in Patients with Liver Cirrhosis
}

\author{
Fawzy Attia*, Khalil A. Khalil, Amira Abdelmonem, and Mohamed Abdel-Fattah
}

Department of Internal Medicine, Faulty of Medicine, Suez Canal University, Ismailia, Egypt

\begin{abstract}
Background: It was found that there is a significant progressive decrease in the serum level of C24 ceramide with increasing severity of cirrhosis. Our aim was to evaluate the efficacy of $\mathrm{C}_{24}$ Ceramide (C24Cer) as a predictor of decompensation in cirrhotic patients at Suez Canal University hospital, Ismailia, Egypt. Subjects and Methods: Patients with viral related liver cirrhosis were consecutively confronted in the outpatient and inpatient sections. They were classified according to Child-Pugh into A, B, C groups. A control group (13 individuals) was compared with each group. Clinical assessment and Liver profile were performed. The serum level of $\mathrm{C} 24 \mathrm{Cer}$ was measured using $20 \mu \mathrm{L}$ extracted serum with methanol: chloroform: $\mathrm{HCl}$ (15:83:2), using liquid chromatography coupled to tandem mass spectrometry. Results: Patients with Child-A cirrhosis showed a significantly higher mean $C 24$ Cer level compared to Child-C patients $(p<0.001)$. Pairwise comparisons showed a statistically significant stepwise decrease in the mean $\mathrm{C} 24 \mathrm{Cer}$ level from control group to Child-A, $B$ then $C$ cirrhotic patients respectively ( $p$-value: $0.029,<0.001$, and $<0.001$ ). There was a highly significant negative correlation between $\mathrm{C} 24 \mathrm{Cer}$ and each of total bilirubin, PT, ascites grade, hepatic encephalopathy grade, and Child score ( $\mathrm{p}<0.001$ for each) as well as a highly significant positive correlation with albumin level $(p<0.001)$. Conclusion: a low serum $\mathrm{C} 24 \mathrm{Cer}$ is associated with hepatic decompensation and is good with a sensitivity of $100 \%$ and a specificity of $73 \%$. Further studies are needed to elucidate the real-life significance of $\mathrm{C} 24 \mathrm{Cer}$ as a non-invasive mortality predictor in cirrhosis.
\end{abstract}

Keywords: liver cirrhosis, Child-Pugh classification, Ceramide 24

\section{Introduction}

Chronic hepatitis $\mathrm{C}$ virus (HCV) infection affects more than 170 million persons worldwide and responsible for the development of liver cirrhosis in many cases $^{(1)}$. Due to the frequently asymptomatic clinical presentation, cirrhosis remains often unsuspected until clinical complications become apparent. Progression of cirrhosis is often preventable and since it is linked with increased mortality rates especially when decompensated, early diagnosis and adequate monitoring of the disease are indispensable ${ }^{(2)}$. The Child-Pugh and Model for End-Stage Liver Disease (MELD) scores are the main clinical tools widely deployed to define short term prognosis of affected patients $(3,4)$, but they do not provide evidence on disease progression and dynamic stage of cirrhosis ${ }^{(5)}$. Incorporation of novel noninvasive markers of disease progression in 
the currently clinically used scores would improve the identification of severely ill patients and enable a more accurate organ allocation in patients with end-stage liver disease. Ceramides are a family of waxy lipid molecules composed of sphingosine and a fatty acid. Ceramides are found in high concentrations within the cell membrane of cells ${ }^{(6)}$. They are one of the component that make up sphingomyelin, one of the major lipids in the lipid bilayer. Contrary to previous assumptions that ceramides and other sphingolipids are found in cell membrane were purely supporting structural elements, ceramide can participate in a variety of cellular signaling, examples include regulating differentiation, proliferation, and programmed cell death (PCD) of cells ${ }^{(6)}$ Roles for ceramide and its downstream metabolites have also been suggested in a number of pathological states including can$\operatorname{cer}^{(7)}$,neurodegeneration ${ }^{(8)}$, obesity associated diabetes ${ }^{(9)}$, microbial pathogenesis and inflammation $^{(10)}$. There is a tight interaction between variations in serum sphingolipid levels and progression of liver fibrosis as well as responsiveness to antiviral therapy ${ }^{(11)}$, particularly, sphingosine, sphinganine, and C24ceramide (C24Cer) that appeared as promising novel biomarkers in chronic HCV infection and should be further evaluated in the context of noninvasive prediction of liver fibrosis ${ }^{(12)}$. A significant decrease of long and very long chain ceramides, particularly of $24 \mathrm{Cer}$ in patients with increasing severity of cirrhosis was found. Additionally, hydropic decompensation, in the form of ascites, significantly correlated to low C24Cer level as well as hepatic decompensation ${ }^{(13)}$. Poor overall survival was observed in those with low serum concentration of $\mathrm{C} 24 \mathrm{Cer}$ as well ${ }^{(13)}$. So, our aim was to evaluate serum level of $\mathrm{C24Cer}$ as a predictor of hepatic decompensation in patients with HCV-related liver cirrhosis in Suez Canal area.

\section{Subjects and Methods}

Patients with HBV and/ or HCV-related liver cirrhosis were consecutively confronted in the outpatient clinic and inpatient department of internal medicine department of the Suez Canal university hospital in Ismailia city and they were classified according to Child-Pugh classification to Child A, B and $C$. A control group of normal population was compared. Accordingly, the study population was divided into 4 groups as follow: Group1: normal population, Group2: Child A, group3: Child B and group 4: Child's C, with each group including 13 patients. Clinical assessment, abdominal ultrasonography, Liver profile, serum creatinine, $C B C$, HCV RNA and HBV-DNA were performed to all groups. Serum level of $\mathrm{C} 24 \mathrm{Cer}$ was investigated using $20 \mu \mathrm{L}$ extracted serum with methanol: chloroform: $\mathrm{HCl}$ (15:83:2). Amount of $\mathrm{C} 24$ was analyzed by liquid chromatography coupled to tandem mass spectrometry. We excluded cirrhotic patients with evidence of malignancy, solid organ transplantation and those with autoimmune disease or on immunosuppressive therapy.

\section{Results}

Our results showed no statistically significant difference in socio-demographic background among various study groups as shown in Tables1\&2. We found a statistically significant difference among the study groups regarding the ceramide 24 level. In Table 3-A; patients with Child-A cirrhosis showed a significantly higher mean $\mathrm{C} 24 \mathrm{Cer}$ level compared to Child-C patients ( $p<0.001)$ as shown in Figure 1. In Table 3-B; Pairwise comparisons showed a statistically significant stepwise decrease in the mean C24Cer level from control group to Child-A, B then $C$ cirrhotic patients respectively (p-: 0.029, <0.001, and <0.001). 
Table 1: Comparison of socio-demographic data among different Child's classes

\begin{tabular}{|l|c|c|c|c|c|c|}
\hline & Control & Class A & Class B & Class C & Total & p-value \\
\hline Age (Mean \pm SD) & $58.46 \pm 9.04$ & $54.92 \pm 3.43$ & $53.77 \pm 9.82$ & $61 \pm 8.73$ & $57.04 \pm 8.42$ & 0.213 \\
\hline Male n (\%) & $3(23.1)$ & $8(61.5)$ & $11(84.6)$ & $8(61.5)$ & $30(57.7)$ & 0.08 \\
\hline Rural residence & $6(46.2)$ & $2(15.4)$ & $8(61.5)$ & $7(53.8)$ & $23(44.2)$ & 0.091 \\
\hline
\end{tabular}

In this study, we found that a low serum C24Cer concentration correlated with a higher frequency of occurrence of hepatic decompensation parameters; as our data showed a highly significant negative corre- lation between $\mathrm{C} 24 \mathrm{Cer}$ and total bilirubin, PT, ascites grade, hepatic encephalopathy grade, and Child score ( $p<0.001$ each) as well as a highly significant positive correlation with albumin level $(p<0.001)$ (Table 4).

\begin{tabular}{|l|c|c|c|c|}
\hline \multicolumn{5}{|c|}{ Table 2: Comparison of the clinical background among } \\
different Child's classes \\
\hline Group* & $\begin{array}{c}\text { Control } \\
\mathrm{N}=13\end{array}$ & $\begin{array}{c}\text { Class A } \\
\mathrm{N}=13\end{array}$ & $\begin{array}{c}\text { Class B } \\
\mathrm{N}=13\end{array}$ & $\begin{array}{c}\text { Class C } \\
\mathrm{N}=13\end{array}$ \\
\hline HBV & 0 & 0 & $2(15.4)$ & $2(15.4)$ \\
\hline HCV & 0 & $10(77)$ & $5(38.5)$ & $8(61.5)$ \\
\hline H. of Bilharziasis & 0 & $1(7.7)$ & $6(46.2)$ & $2(15.4)$ \\
\hline Hypertension & $1(7.7)$ & $1(7.7)$ & $1(7.7)$ & $1(7.7)$ \\
\hline Diabetes mellitus & 0 & $1(7.7)$ & $2(15.4)$ & $3(23.1)$ \\
\hline Pregnancy & $1(7.7)$ & 0 & 0 & 0 \\
\hline Other disorders & 0 & 0 & 0 & $3(23.1)$ \\
\hline
\end{tabular}

Figure 2 shows a scatterplot for ceramide 24 level in relation to both serum albumin and total bilirubin levels. In addition, the univariate analysis revealed a significant association between a low serum level of C24Cer and the presence of ascites $(p<0.001)$ and hepatic encephalopathy $(p=0.003)$ as shown in Table 5 . While Figure 3 shows the ROC curve parameters of hepatic decompensation in relation to serum ceramide 24 level. It shows that serum ceramide 24 level can be used as a good indicator of hepatic decompensation with a sensitivity of $100 \%$ and specificity of $71.8 \%$. Our results showed that 24 Cer can be used as a good indicator for hepatic decompensation with a sensitivity of $100 \%$ and a specificity of $73 \%$.

\begin{tabular}{|c|c|c|c|c|}
\hline \multicolumn{5}{|c|}{ Table 3A: Comparison of the mean serum C24Cer level among different Child's classes } \\
\hline Group & Control & Class A & Class B & Class C \\
\hline & mean \pm SD & mean \pm SD & mean \pm SD & mean \pm SD \\
\hline C24Cer level & $6417.54 \pm 2451.41$ & $3506.69 \pm 150.57$ & $2132.69 \pm 123.27$ & $1126.85 \pm 87.28$ \\
\hline
\end{tabular}

A significant difference was found among the study groups regarding C24Cer level. (<0.001)

\section{Discussion}

We aimed in this study at assessing the serum concentration of $\mathrm{C} 24$ ceramide in a se- ries of patients with different stages of liver cirrhosis as well as evaluating its predictive potential regarding disease progression, hepatic decompensation, and overall 
survival. We found that patients with ChildA cirrhosis showed significantly higher concentration of ceramide 24 compared to those with Child-C ( $p$ <0.001). Pairwise comparisons of category regarding ceramide 24 showed a statistically significant difference between Child- $C$ cirrhotic patients and each of Child-B, Child-A patients, and controls $(p=0.029,<0.001$, and $<0.001$ respectively).

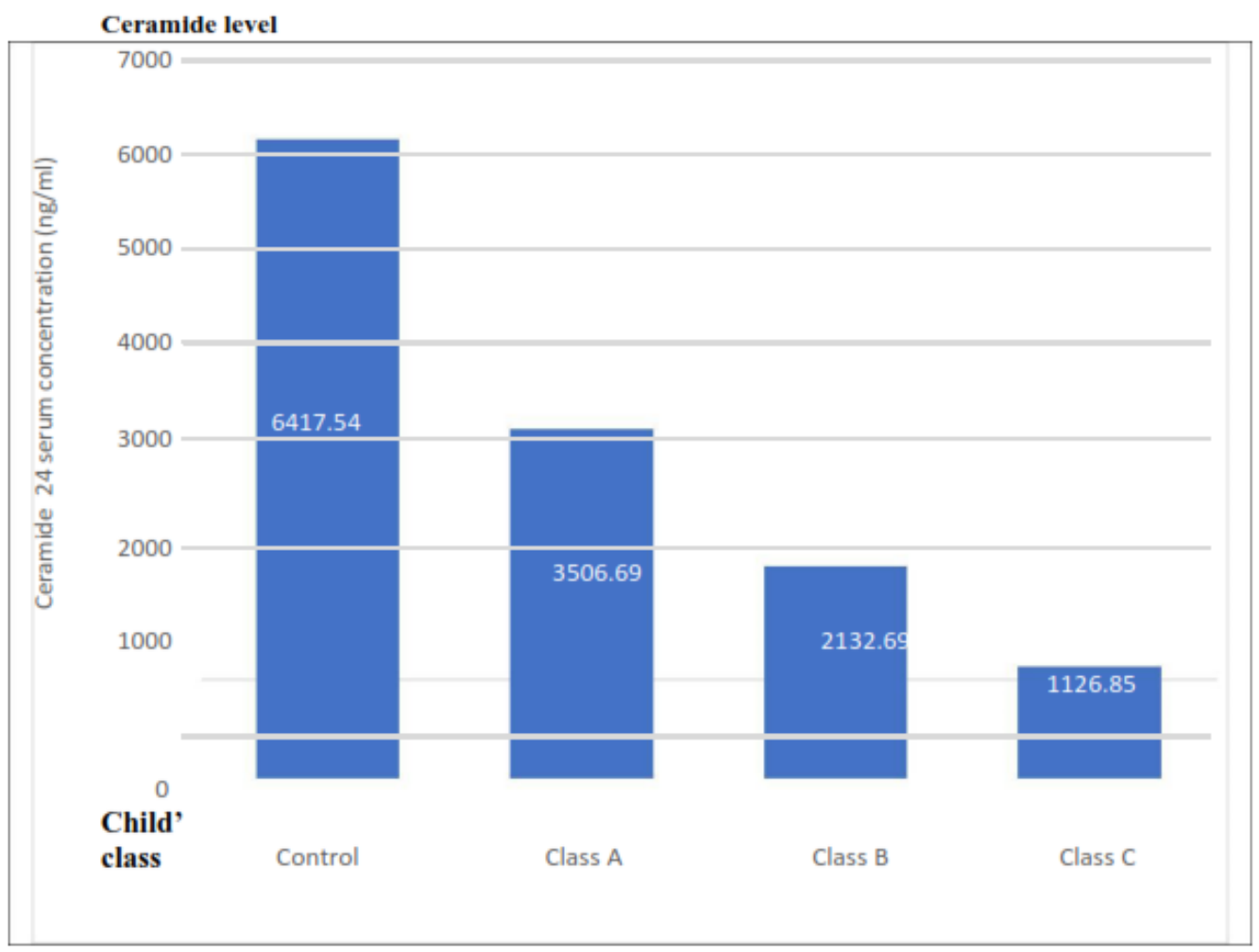

Figure 1: Comparison of the mean Ceramide 24 level according to the Child class

These results were consistent with previous studies. For instance, in their study to evaluate serum sphingolipid profile of 244 patients with cirrhosis prospectively followed for a median period of $228 \pm 217$ days via mass spectrometry, Grammatikos et al ${ }^{(13)}$ demonstrated that patients with Child-A had significantly higher concentrations of long and very long chain Cer's compared to patients with Child-C cirrhosis $(p<0.001)$.

\begin{tabular}{|c|c|c|}
\hline \multicolumn{3}{|c|}{ Table 3B: Pairwise comparisons of different Child's } \\
classes regarding C24Cer
\end{tabular}


Furthermore, comparisons between Child-B cirrhosis and Child-A or Child-C cirrhosis revealed marked differences as well. In the current study there was a significant variation of serum C24Cer levels observed in patients with cirrhosis caused by $\mathrm{HCV}$ $(p=0.017)$ which is similar to the results of the previous studies. One study which compared 72 healthy volunteers to 69 patients with non-alcoholic fatty liver disease and 69 patients with chronic hepatitis C virus infection identified $\mathrm{C} 24 \mathrm{Cer}$ being significantly decreased in chronic HCV infection as compared to healthy individuals and patients with NAFLD ${ }^{(12)}$. Similarly, another study demonstrated a significantly decreased serum C24Cer in patients with HCVinduced liver cirrhosis as compared to cirrhosis induced by other chronic hepatopathies ${ }^{(8)}$. Moreover, in this study we found that low serum Cer concentration correlated with higher rates of hepatic decompensation; as a significant strong negative correlation was found between serum ceramide 24 and total bilirubin, PT, ascites, hepatic encephalopathy grade, and Child score ( $p<0.001$ for each respectively) as well as a significant strong positive correlation was found with serum albumin level $(p<0.001)$.

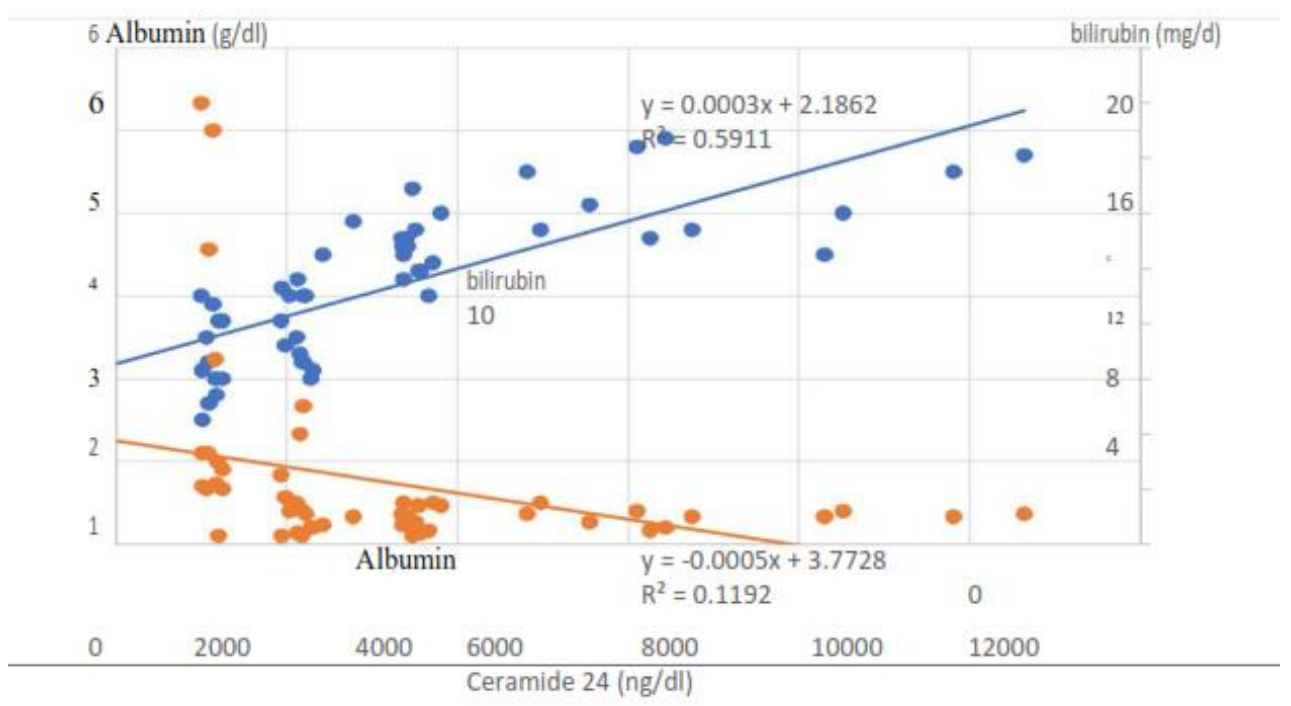

Figure 2: Correlation of the serum Ceramide 24 level with both serum total bilirubin and albumin

Additionally, the univariate analysis revealed a significant association between the serum C24Cer and ascites $(p<0.001)$ as well as hepatic encephalopathy $(p=0.003)$. In line with these findings, the study of Grammatikos et al. demonstrated decreased serum concentrations of long and very long chain ceramides associated with the presentation of major clinical complications defining hep- atic decompensation such as ascites, spontaneous bacterial peritonitis, hepatic encephalopathy and hepatorenal syndrome with ascites occurrence being only associated with serum concentration of 24 Cer and dhC24Cer ( $p<0.001$ and $p<0.01$ respectively). Another study demonstrated a decreased $\mathrm{C} 24 \mathrm{Cer}(\mathrm{P}=0.028)$ were associated with severe liver fibrosis $\left(\mathrm{F}_{3}-\mathrm{F}_{4}\right){ }^{(7)}$.

\begin{tabular}{|c|c|c|c|c|c|c|}
\hline \multicolumn{7}{|c|}{ Table 4: Correlation between serum Ceramide 24 and different parameters of } \\
hepatic decompensation in the study population \\
\hline Variable & Child's grade & S. Bil. & S. Alb. & PT & H.E. grade & Child's Score \\
\hline Ceramide 24 (R) & -0.93 & 0.520 & 0.834 & -0.738 & -0.549 & 0.887 \\
\hline
\end{tabular}


In another study, assessing variations in serum sphingolipid levels associated with liver fibrosis progression and poor treatment outcome in $\mathrm{HCV}$, it was observed serum C24Cer associated significantly with severity of liver fibrosis in HCV patients ${ }^{(10,11)}$. Additionally, when included in a multivariate lo- gistic regression model after adjustment for other variables, also in univariate analysis, it is associated with liver fibrosis progression within the HCV patient cohort. Our results showed that serum C24Cer can be used as a good indicator for hepatitis infection with a sensitivity of $100 \%$ and specificity of $71.8 \%$.

\begin{tabular}{|l|c|}
\hline \multicolumn{2}{|c|}{$\begin{array}{c}\text { Table 5: Univariate analysis of Different varia- } \\
\text { bles in association with serum ceramide 24 }\end{array}$} \\
\hline Variable & P value \\
\hline- Sex & 0.024 \\
\hline- HCV & 0.017 \\
\hline- DM & 0.018 \\
\hline- Bilirubin & 0.002 \\
\hline- Albumin & $<0.001$ \\
\hline- PT & 0.001 \\
\hline- Ascites & $<0.001$ \\
\hline- HE & 0.003 \\
\hline- Score & $<0.001$ \\
\hline- Hepatitis & $<0.001$ \\
\hline
\end{tabular}

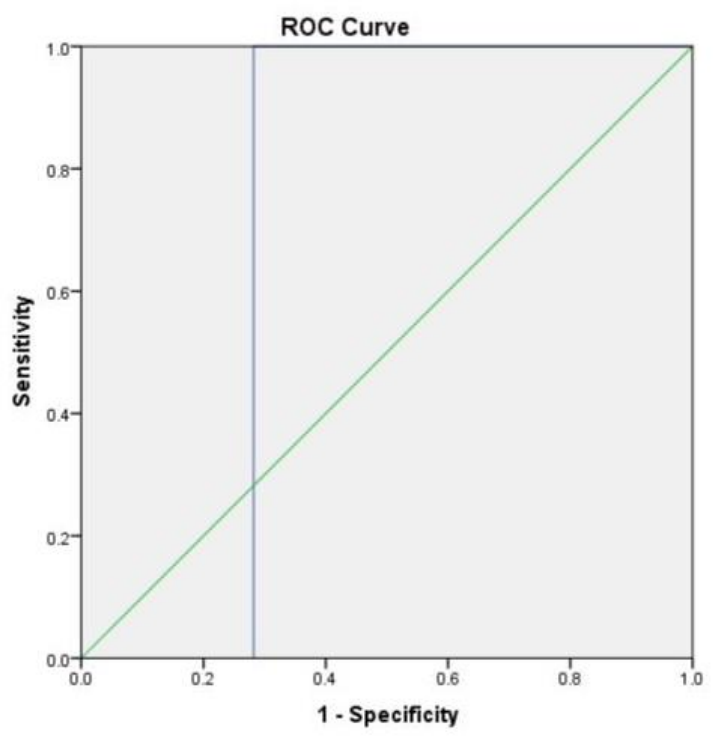

\begin{tabular}{|c|c|c|c|c|c|c|c|c|c|c|}
\hline Area & P-value & Cut-off point & Sn & Sp & PPV & NPV & LR+ & LR- & DOR & A \\
\hline 71.8 & $\mathbf{0 . 0 2}$ & 3068.5 & 100 & 71.8 & 33 & 15.4 & -1.41 & -1.38 & 1.02 & 28.8 \\
\hline
\end{tabular}

Figure 3: ROC curve of the serum ceramide 24 level as an indicator hepatic Decompensation 
Additionally, it can be used as an indicator for decompensation with a sensitivity and specificity of $100 \%$. Despite all these promising findings, this study has some limitations, as a clinical association study, it is difficult to ascertain causation and to establish the causal relationship between SL metabolism and cirrhosis. Thus, future prospective cohort studies are suggested to better understand this relationship. In addition, data regarding the survival are lacked in our study and they could have been beneficial to effectively assess the predictive ability of the serum C24Cer of survival. Moreover, since serum Cer's are bound to lipoproteins such as low-density lipoprotein (LDL), high density lipoprotein (HDL) and very low-density lipoprotein (VLDL) it cannot be excluded that the observed differences are due to changes of the mentioned lipoproteins in patients with cirrhosis. Unfortunately, serum levels of these lipoproteins were not available in our patients.

\section{Conclusion}

our study identified that $\mathrm{C} 24 \mathrm{Cer}$ is associated with progression of the hepatic decompensation. Certainly, further studies are needed to elucidate the underlying mechanism as well as the role of serum SL's and particularly $\mathrm{C} 24 \mathrm{Cer}$ in the non-invasive mortality prediction in patients with cirrhosis.

\section{References}

1. Rockey DC, and Friedman SL (2012): Hepatic Fibrosis and Cirrhosis. Hepatology 6485.

2. Lim YS and Kim WR (2008): The global impact of hepatic fibrosis and end-stage liver disease. Clin Liver Dis 12: 733-46.

3. Christensen E, (2004): Prognostic models including the Child-Pugh, MELD and Mayo risk scores - Where are we and where should we go?" J Hepatol 41: pp. 344-50.
4. Malinchoc M, Kamath PS, Gordon FD, et al., (2000): A model to predict poor survival in patients undergoing trans-jugular intrahepatic portosystemic shunts," Hepatology 31: 864-71.

5. Udompap P, Kim D and Kim WR (2015): Current and Future Burden of Chronic Nonmalignant Liver Disease. Clin. Gastroenterol. Hepatol 13: 2031-41.

6. Bikman BT and Summers SA (2011): "Ceramides as modulators of cellular and whole-body metabolism," J Clin Invest, 121: 4222-30.

7. Hartmann D, Lucks J, Fuchs $S$, et al. (2012): "Long chain ceramides and very long chain ceramides have opposite effects on human breast and colon cancer cell growth," Int J Biochem Cell Biol. 44:620-28.

8. Van Echten-Deckert $G$ and Walter J, (2012): "Sphingolipids: critical players in Alzheimer's disease." Prog. Lipid Res., 51: 378-93.

9. Haus JM, Kashyap SR, Kasumov T, et al. (2009): "Plasma ceramides are elevated in obese subjects with type 2 diabetes and correlate with the severity of insulin resistance," Diabetes, 58:337-43.

10. Lightle S, Tosheva R, Lee A, et al. (2003): "Elevation of ceramide in serum lipoproteins during acute phase response in humans and mice: role of serine-palmitoyl transferase," Arch. Biochem. Biophys, 419:120-8.

11. Grammatikos G, Ferreiros N, Bon D, et al., (2015): Variations in serum sphingolipid levels associate with liver fibrosis progression and poor treatment outcome in hepatitis $C$ virus but not hepatitis $B$ virus infection. Hepatology. 61(3):812-22.

12. Grammatikos $\mathrm{G}$, Mühle C, Ferreiros N, et al. (2014): Serum acid sphingomyelinase is upregulated in chronic hepatitis $C$ infection and non- alcoholic fatty liver disease. Biochim Biophys Acta-Mol Cell Biol Lipids. 1841 (7):1012-20.

13. Grammatikos G, Ferreiròs N, Waidmann O, et al. (2015): Serum Sphingolipid Variations Associate with Hepatic Decompensation and Survival in Patients with Cirrhosis. Cowart A, PLoS One. 10(9):130-8. 